\title{
«Il ne faut pas / Que le bon menestrier accorde / Toujours son chant sur une corde »: Shakespeare, Ronsard et la polyphonie
}

Agnès Lafont

\section{(2) OpenEdition \\ 12 Journals}

\section{Édition électronique}

URL : http://journals.openedition.org/shakespeare/1056

DOI : $10.4000 /$ shakespeare.1056

ISSN : 2271-6424

\section{Éditeur}

Société Française Shakespeare

\section{Édition imprimée}

Date de publication : 1 novembre 2007

Pagination : 213-227

ISBN : 2-9521475-3-1

\section{Référence électronique}

Agnès Lafont, « «II ne faut pas / Que le bon menestrier accorde / Toujours son chant sur une corde » : Shakespeare, Ronsard et la polyphonie », Actes des congrès de la Société française Shakespeare [En ligne], 24 | 2007, mis en ligne le 30 mars 2010, consulté le 06 mai 2019. URL : http://

journals.openedition.org/shakespeare/1056 ; DOI : 10.4000/shakespeare.1056

Ce document a été généré automatiquement le 6 mai 2019.

(c) SFS 


\title{
«Il ne faut pas / Que le bon menestrier accorde / Toujours son chant sur une corde ": Shakespeare, Ronsard et la polyphonie
}

\author{
Agnès Lafont
}

1 L'Adonis (1564) et les Vers d'Eurymedon et de Calliree (1573) de Ronsard et le Vénus et Adonis de Shakespeare ${ }^{1}$ offrent trois versions de la fable ovidienne (Métamorphoses, $\mathrm{x}, 345 \mathrm{sq}$ ). Dans ces poèmes, le motif mythologique pose une question sur la sincérité de l'amour pétrarquiste; qu'il soit fidèlement repris (Adonis) ou subverti (Venus and Adonis présente une lecture totalement originale avec un Adonis rebelle à l'amour, chasseur intrépide, plus proche de la figure amazonienne d'Hippolyte) ou encore irradiant (dans Les Vers d'Eurymedon et de (alliree), le canevas mythologique commente les artifices de la rhétorique amoureuse pétrarquiste. D'un point de vue purement formel, ces poèmes présentent une tension interne qui traduit un certain détachement: tous trois se complaisent à la variété des tons, apportant une note d'originalité à la thématique rebattue des aventures amoureuses. L'expression mythologique est précieuse pour faire ressortir la critique des conventions poétiques.

2 Il s'agit moins de s'attarder sur le contenu thématique du mythe et de ses interprétations que de s'interroger sur le volontaire mélange des tons présents dans ces trois pièces poétiques. La citation de Ronsard, dans "Le Fourmy (Le Bocage, 1554), propose un véritable art poétique à l'usage de la variété des tons :

Aussi toujours, il ne faut pas

Que le bon menestrier accorde

Toujours son chant sur une corde.

Et qui voudra bien plaire, il faut

Ne chanter pas toujours le haut. (v. 18-22)

3 Le jeu sur la répétition de "toujours / pas toujours " montre, sur un ton détaché, combien la variation est nécessaire à la composition; à un autre niveau, la Vénus 
shakespearienne commente l'émoi amoureux en des termes extatiques qui livrent aussi une réflexion méta-textuelle :

Melodious discord, heavenly tune harsh sounding

Ears' deep sweet music, and heart's deep sore wounding

$(V \& A, 431-432)^{2}$

Oxymores et parallélismes lient comble d'amour et de douleur en suivant la métaphore musicale du contrepoint, autre lecture des effets de la varietas. Elle permet de lier les contraires tout en maintenant leur incompatibilité.

La fiction tirée des Métamorphoses semble gloser avec un certain détachement (voire avec ironie) la description pétrarco-platonicienne d'un amour en tension, restée irrésolue, entre l'aspiration vers l'idéal et la préférence pour les plaisirs plus mondains. Il s'agit de montrer que «l'écart» entre nos textes et leurs intertextes n'est pas le fruit de la juxtaposition servile de plusieurs sources mais le résultat d'une combinaison destinée à les faire se commenter entre eux. Certaines convergences troublantes apparaissent alors : l'allusion mythologique éclaire le contraste, inhérent au pétrarquisme, entre l'idéal amoureux et la consommation charnelle rêvée. Les poèmes semblent mettre en conversation le mythe ovidien avec l'expression de l'amour, explorant les ambivalences qui étaient celles de l'expérience pétrarquéenne ${ }^{3}$.

6 La polyphonie induit l'ironie sur le plan purement narratif. On est là dans le domaine de la mythologie burlesque qui permet des effets comiques. Ainsi, dans le poème de Ronsard, Adonis (1564), la juxtaposition entre récit de la mort du jeune chasseur et complainte de Vénus crée un détournement amusant. Ce poème résulte, en effet, de l'interpénétration entre la métamorphose ovidienne, une idylle de Bion et des fragments d'une églogue de Navagero. La tonalité de ce long poème narratif qui pourrait n'être qu'une traduction / adaptation du texte des Métamorphoses semble de prime abord à visée édifiante, comme le laisse entendre l'adresse au lecteur :

Vien lire de Vénus le bien et le malheur

Car toujours un plaisir est meslé de douleur (3-4)

7 Après la narration des parfaites amours filées par les amants, interviennent les lamentations de Vénus qui durent 153 vers durant lesquels agonise longuement le malheureux chasseur. Un décalage naît entre, d'une part, la tonalité grandiloquente de la cascade de plaintes de Vénus, directement inspirée du texte de l'idylle de Bion dont Ronsard reprend le refrain : « Hélas pauvre Adonis, tous les amours te pleurent / Par ta mort, Adonis, toutes delices meurent!» à neuf reprises en crescendo, avec quelques variations sur le second vers du distique, et, d'autre part, la situation mythologique «adaptée ». Le comique de cette agonie prolongée du jeune homme, témoin des manifestations exagérées de la peine de son amante, est fortement souligné par la brève chute du récit qui montre que, sitôt mort, il est oublié (en cinq vers). L'idylle de Bion est enchâssée dans la narration mythique déstructurée, ralentie puis accélérée, dès lors présentée comme une littérature convenue du tourment amoureux.

8 Le second poème de Ronsard que nous étudions propose une variante amusante de l'échange de l'arc de Cupidon avec celui de Diane, topos marotique heureusement mis au service du commentaire parodique. Les Vers d'Eurymédon et de Callirée est une fresque mythologique composite de l'invention de Ronsard ${ }^{4}$, faite sur le «commandement des grands ${ }^{5}$ ", ce qui a été bien analysé comme une allégorisation courtisane de l'aventure de Charles IX avec Anne d'Atri d'Acquaviva. Ainsi, le jeu sur le nom de la nymphe des eaux vives, d'Acquaviva, sert de nombreuses allusions galantes aux contrariétés de l'eau 
apaisante et du feu du désir, sur un mode tout pétrarquiste. Quittant le domaine de la lecture d'une mythologie de cour dite "à clef ", nous souhaitons ici interroger la variété des tons dans ce recueil qui propose une unité narrative disloquée qui n'est pas sans rappeler la poétique de la fragmentation qui régit le Canzoniere de Pétrarque.

Nous avons là six poèmes qui appartiennent à cinq genres ou formes différentes : tantôt, dans les Stances I, l'amant chasseur témoigne de son amour en sacrifiant aux artifices pétrarquistes; tantôt, en une analepse explicative, le poète donne à voir l'innamoramento des amants et l'explique par un mythe "sur mesure ", sur le mode parodique; tantôt encore, Eurymédon parle et, dans la pièce en forme d'Idylle intitulée «Le baing de Callirée ", permet au lecteur une plongée dans son esprit tourmenté par le désir sensuel de connaitre Callirée ; c'est au Poète lui-même de prendre, ensuite, la parole dans une Élégie qu'il adresse à Eurymédon; Eurymédon lui répond en une Chanson par Stances qui reprend le motif aimable de l'aimée comme une onde vive dans laquelle il souhaite se plonger. Dans le sonnet conclusif, c'est enfin au tour de Callirée de confier son angoisse et sa peur de l'accident de chasse qui pourrait menacer son amant, « nouvel Adonis ». Dans les deux premières Stances, la variété des tons permet d'opposer déclaration amoureuse convenue et fiction mythologique sur le mode parodique. L'éloquence platonicienne de l'amant semblait plaider la légèreté de son pur amour :

Si vous aimiez autant vous seriez plus légère (66)

Ainsi dedans le ciel mon corps qui n'est plus mien

Alembiqué d'amour, s'envole de nature (47-48)

10 Mais le jeu de mots sur la légèreté amoureuse n'est pas loin! Le changement brutal de registre entre les deux premières Stances, qui veut qu'à la plainte pétrarquiste succède la verve héroï-comique des chassés-croisés de Diane et de Cupidon, alerte le lecteur sur la prise de distance qui s'opère. À l'intensité des antithèses passionnées et des serments amoureux succèdent, au sens propre, les échanges des divinités tutélaires :

Amour sera sans arc, sans trait et sans brandon

Et tout sera changé plutôt qu'Eurymédon

Oublie les amours qu'il porte à Callirée (Stances I, 94-96)

11 Dans les Stances suivantes, le début de la fiction s'annonce comme une variation sur cette métaphore attendue qui est alors prise au pied de la lettre :

De fortune Diane et l'Archerot Amour

En un mesme logis arriverent un jour,

L'un lassé de voler, et l'autre de la chasse :

Destendirent leurs arcs et pour prendre repos,

Leurs carquois pleins de traicts deschargerent du dos,

Et les mirent ensemble en une mesme place. (1-6)

Qu'arriva-t-il?

[...] Diane au poinct du jour soigneuse s'éveilla,

Et pour tromper Amour usa de diligence :

Print son arc pour le sien, ses feux et son carquois,

Puis se mocquant de lui s'en alla par les bois,

Desireuse de faire belle vengeance [...]. (7-12)

Pire: "Diane se transforme / en l'image d'Amour", utilisant une nymphe des eaux, Callirée, pour faire succomber Eurymédon. La voix du mythe produit ainsi trois effets: d'abord, la reprise des attributs de Cupidon induit un effet d'écho et de transition entre les deux Stances; ensuite, se produit un effet de variation voire d'inversion puisque c'est Diane qui va infliger la flèche du tourment amoureux ${ }^{6}$. Ronsard travaille ici à partir du 
topos marotique ${ }^{7}$ de l'inversion entre la chasse de Diane et celle de Cupidon. Le déguisement de Vénus en Diane lorsqu'elle suit Adonis à la chasse, donné par Ovide, est repris et retravaillé de manière ironique pour gloser l'inconstance de l'amour alors même qu'il est question d'expliquer le mythe fondateur de cet innamoramento. Enfin, c'est le troisième effet, le changement de registre fait que le mythe ainsi créé vient commenter l'ensemble des Vers d'Eurymédon et de Callirée en induisant un doute sur la durée des sentiments des amants. La marqueterie de la composition intègre avec virtuosité la topique de la plainte amoureuse dans l'anecdote mythologique inventée, introduisant l'ironie, « l'écart » par le recours à la fable.

Le troisième exemple nous est fourni par Venus and Adonis. Étudier les sources d'inspiration de la création shakespearienne dans le corpus des Métamorphoses ${ }^{8}$ permet de suggérer que l'origine du renversement des rôles sexuels dans Venus and Adonis proviendrait d'une contamination par la très populaire fable de Salmacis et Hermaphrodite. Cette contamination permet l'effet immédiatement parodique du mythe qui prend à rebours la posture traditionnelle de la belle dame insensible, chère aux cœurs des amants-poètes pétrarquistes :

Being so enraged, desire doth lend her force

Courageously to pluck him from his horse

Over one arm the lusty courser's rein,

Under the other was the tender boy,

Who blushed and pouted in a dull disdain

With leaden appetite, unapt to toy. (29-34)

Utilisant un comble mythologique burlesque, l'intention satirique s'anime grâce au scénario fabuleux ainsi ré-arrangé. La variété mythologique due à une "copieuse " hybridation témoigne en fait d'une relecture critique de la fable qui la transforme en un commentaire qui revivifie le topos amoureux. L'écart dégonfle la convention pétrarquiste des rôles masculin et féminin dans l'attirance amoureuse. Tel est le rôle du mythe: articuler la diversité des styles (comique, folâtre ou élégiaque). Le discours antipétrarquiste, loin de récuser des ornements poétiques qui seraient jugés artificiels, utilise le mythe pour moquer le manque de sincérité et pour avouer la puissance de la libido. Varier son style, c'est, en quelque sorte, le métamorphoser au cours du poème : or le mythe de la métamorphose, voire de la transformation, attire l'attention du lecteur sur la façon dont le contenu mythique est exploité. Derrière l'intérêt narratif, c'est l'intention rhétorique qu'il faut percevoir.

Voyons, en premier lieu, comment l'hybridation des lectures mythiques permet la variété ; dans les Vers d'Eurymédon et de Callirée, glose morale et lecture galante insufflent une nouvelle vie au mythe de l'hermaphrodite, jouant des ambiguités sexuelles. Rappelons que Cassandre se déguise d'abord en Vénus puis en Adonis dans Les Amours de 1552, sonnet 86 :

Quel plaisir est-ce, ainçois quelle merveille,

Quand ses cheveux troussez dessus l'oreille,

D’une Vénus imitent la façon?

Quand d'un bonnet sa teste elle Adonise

Et qu'on ne sçait s'elle est fille ou garçon,

Tant sa beauté en tous deux se déguise? (Pl. I, p. 71-72)

17 Alors que l'Adonis de Shakespeare, frêle éphèbe, est le jouet d'une mégère amoureuse, l'Eurymédon de Ronsard souhaite se changer : 
[...] d'homme en femme, ainsi que fit Cœnée ${ }^{9}$

Cœnée qui tournant par miracle sa peau

Estoit tantôt pucelle, et tantôt jouvenceau (« Le baing de Callirée », 2-4)

Dans l'ensemble du recueil, deux mythèmes sont retenus de la fable de l'hermaphrodite : celui de la métamorphose (qui revient dans chacune des pièces poétiques comme un lien de composition suggéré) mais surtout le motif fabuleux du Bain de la Nymphe: le " carrefour mythique ${ }^{10}$ » fait ici se rencontrer Vénus et Adonis à la fontaine d'une Dame, autre Cythère, fantasmée comme une accueillante Salmacis qui ouvrirait ses bras à l'amant timide. Le mythe qui affleure ici, derrière celui de Cénée, est celui de Salmacis, traditionnellement lu comme une mise en garde contre les mœurs efféminées, transformé de manière galante et ludique; le renversement est qu'ici c'est le jeune homme qui s'imagine femme et non la femme qui devient Hermaphrodite :

Si j'avais pour jouyr de chose tant aimée

Pour ce jour ma nature en femme transformée,

Je pourrois sans vergongne à son baing me trouver (« Le baing de Callirée », 21-23)

La présence menaçante de l'accident de chasse (qui rejoue au niveau tragique l' innamoramento des amants) introduit la thématique de la dangereuse "vergongne " condamnant Actéon qui a surpris Diane au bain. La liberté du traitement mythologique n'est pas ce qui nous intéresse de prime abord mais elle vient illustrer «l'irrévérence » et la liberté de ton du Ronsard qui se fait mythologue.

En outre, pour Terence Cave, l'intervention de ce mythème du Bain de la Déesse « désorganise la structure thématique pour répondre à une intention rhétorique (donc située en profondeur dans la fabrication de l'œuvre ${ }^{11} »$ : il s'agirait de perdre son identité sexuelle pour explorer les structures verbales du corps de Callirée (Terence Cave, op. cit., p. 245). Cette rupture rhétorique est liée à un contenu mythologique qui vient gloser librement le schéma amoureux de Vénus et Adonis, tel qu'il est hérité des Métamorphoses. Le fabuleux rêve éveillé d'Eurymédon reflète à un autre niveau l'écriture fabuleuse de Ronsard, commentant ainsi son inventio et sa formule de composition puisqu'il s'agit d'un mythe de métamorphose. Le cliché pétrarquiste (la souffrance face à une femme belle et inaccessible), véhiculé par l'exemplum d'Actéon, si en vogue dans ces années-là ${ }^{12}$, est remis en question par sa rencontre avec la fiction mythologique du Bain qui libère l'imaginaire poétique et sensuel de l'amant - et du poète.

Dans Venus and Adonis, le récit de la libération du cheval d'Adonis offre un fonctionnement qui nous semble analogue. Vénus pervertit la rhétorique exemplaire par un intertexte mythique, en rappelant le destrier de Mars : la modification ironique naît du va-et-vient entre le tableautin, enchâssé dans la suasio de l'amoureuse, et la confrontation avec le mythe sous-jacent de Mars et Vénus. En effet, l'image du destrier de Mars, attaché à un arbre, sellé par un Cupidon qui s'interpose entre le dieu de la guerre et son épée, est un motif iconographique typique qui symbolise la retenue des passions, lecture habituelle de l'amour entre Mars et Vénus. Toutefois, Vénus interprète différemment ce symbole au moment où l'étalon se libère pour rejoindre la jument :

How like a jade he stood tied to the tree,

Servilely mastered with a leathern rein;

But when he saw his love, his youth's fair fee,

He held such pretty bondage in disdain,

Throwing the base thong from his bending crest,

Enfranchising his mouth, his back, his breast. (391-396) 
plaide pour la libération des passions, contredisant la rhétorique emblématique habituelle de l'exemplum ${ }^{13}$. Son traitement léger de l'anecdote modifie sensiblement la portée parénétique de l'argument traditionnel :

Look what a horse should have he did not lack,

Save a proud rider on so proud a back (299-300 $)^{14}$

La description personnifiée du cheval, travesti en «melancholy malcontent » (v.313), le type même de l'amoureux éconduit des années 1590, ajoute encore à ce traitement parodique. Si la longueur du passage, la description minutieuse de l'animal composent une saynète statique dans la narration et offre une clef interprétative pour comprendre, à un autre niveau, le comportement débridé de la déesse de l'amour, si le renversement des rôles (destrier/jument) par rapport à ce qui se joue dans l'action englobante (Vénus/ Adonis) crée un décalage fructueux, il faut toutefois noter que les échos étouffés du mythe des amours harmonieuses de Mars et Vénus ${ }^{15}$ viennent offrir un contrepoint intéressant à une lecture qui condamnerait la passion amoureuse comme force lascive irrésistible. Les différentes voix, celle de l'exemplum, celle de la fable, celle de Vénus, contribuent à laisser planer une certaine indécision sur le statut de l'amour passion. C'est ce que rappelle Colin Burrow dans son édition récente des Complete sonnets and poems: « in fact the episode artfully contributes to the poem's careful indecision about whether passion is an unbridled force which can carry you away, or a natural instinct to which Adonis is perversely resistant » (p. 29). Ici encore, le mythe permet de questionner la relation amoureuse en confrontant diverses voix qui dialoguent par l'imagination poétique sans imposer de conclusions.

Sur le mode de la copia, la variété des réfections mythologiques ${ }^{16}$ permet un commentaire sur « l'art de pétrarquiser ${ }^{17}$ ». L'intention rhétorique de cette métamorphose poussée à l'extrême est servie par une invention mythologique personnelle: celle-ci puise à la source de deux traditions, pétrarquiste et ovidienne, pour gloser la méthode de composition des auteurs. Au changement des contenus correspond le changement des formes (avec l'utilisation de la fable, de l'idylle, de la complainte...). À un autre niveau, c'est dans la multiplicité des formes que se donne à entendre une polyphonie qui illustre la liberté de l'auteur, un style. De cette esthétique bigarrée, polymorphe, naît une forme nouvelle, libérée, qui utilise la fable et sa structure métamorphique pour commenter sa propre écriture.

La prolifération "copieuse", dans ces trois pièces poétiques, se fait sur le mode d'un continu allégué qui révèle cependant des failles, des intermittences dans le texte. Tout d'abord, on voit que la variété générique engendre un effet de discontinu. Chez Ronsard, dans Adonis, le souci de la cohérence narrative dans l'inventio mythique conduit, pourtant, à l'échange de la fable ovidienne enchâssée d'Atalante et Hippomène par une réécriture originale de la jalousie de Mars à l'encontre d'Adonis ; c'est que Ronsard privilégie la " cohérence narrative " dans son poème : supprimant la digression que représente la fable d'Atalante et Hippomène chez Ovide, il met en scène Mars, amoureux éconduit, demandant le secours de Diane pour servir sa vengeance ${ }^{18}$. L'invention ronsardienne procède par réfection mythique : autre Vulcain, Mars demande un accident de chasse pour assouvir sa jalousie. L'intervention délienne est appelée par l'allusion au sanglier, associé au monstre Calydonien :

Eslance au devant de ses yeux

Digne d'un Meleagre, un sanglier furieux (II, v. 167-168) 
refection fonctionne sur le mode de la copia, qui permet aux auteurs d'associer sur le mode énumératif des motifs topiques, par figure, pourrait-on dire. Processus mécanique, procédé rhétorique d'école ? C'est l'effet qui compte : Ronsard «affirme son originalité en remplaçant cette insertion par un épisode de son crû ${ }^{19}$ ». De surcroît, à l'innovation mythologique fait écho l'insertion d'un genre, l'hymne, dans un autre, l' epyllion. Rappelons que Terence Cave, dans Cornucopia, considère cette contamination des genres comme un trait caractéristique de la poétique ronsardienne. Mars compose un hymne en miniature à Diane sur le schéma des Hymnes de 1555 : nomination et définition de la divinité, sa louange, description de ses attributs, épisode plus ou moins développé de sa légende, affirmation de l'efficacité de ses interventions (Mars rappelle ici son intervention salvatrice auprès de Diane menacée de viol par Orion) ${ }^{20}$. Variété mythologique et variété générique sont alors totalement mises au service de la continuité de la voix narrative.

Totalement? Non, car le réseau textuel des allusions mythologiques, s'il procède par assemblages, par réfection, dans un souci de cohérence entre mythe et écriture de l'amour, fonctionne à un autre niveau comme du discontinu : le mythe, par le biais de sa glose, autre voix qui vient se juxtaposer à la continuité du poème, dans l'Adonis de Ronsard permet un commentaire ironique sur la situation amoureuse pétrarquiste idéale qui était décrite. La tonalité pseudo- moralisante se trouve contredite par la fin épigrammatique du récit. Sous une forme lapidaire, la pointe bien placée renouvelle le topos du tempus fugit (la fuite du temps) et celui du carpe diem (plaisir de l'instant).

Si tost qu'ell'le vit mort, Amour d'autre costé

Luy a plus tost que vent son regret emporté,

Si qu'elle qui estoit n'agueres tant esprise

D’Adonis, l'oublia pour aimer un Anchise. (Pl. II, p. 322-323, v. 359-362)

la fleur, signe du caractère éphémère de la beauté qu'incarne Adonis, est détournée pour s'appliquer à l'inconstance proverbiale des amours féminines.

Telles sont et seront les amitiez des femmes,

Qui au commencement sont plus chaudes que flames:

Ce ne sont que souspirs, mais en fin telle amour

Ressemble aux fleurs d'Avril qui ne vivent qu'un jour. (Pl. II, p. 323, v. 365-368)

Derrière la voix du mythe se fait entendre une autre voix, insistante : celle de la glose narquoise. Cette insertion $d u$ mythe dans la texture du poème, qui lui permet un commentaire rhétorique, et non plus seulement thématique, il faut aussi la chercher dans le traitement du mythe de Narcisse. Dans la Chanson par Stances (Vers d'Eurymédon et de Callirée, pièce poétique v) l'amant souhaite se métamorphoser en eau car Callirhoe signifie le « beau ruisseau ». Quelle que soit la généalogie de Callirée (fille d'Océan et de Téthys, fille du dieu du fleuve Acheloos ou encore fille de Scamandre), elle est la « belle eau vive » à laquelle il veut se mélanger, pour se fondre en elle. L'évocation du mythe de Narcisse par Vénus, dans Vénus et Adonis, relève d'une rhétorique de l'exemplum, mettant en garde contre la stérilité :

Narcissus so himself himself forsook

And died to kiss his shadow in the brook (161-162) ${ }^{21}$

Celle-ci subit en revanche un détournement aimable chez Ronsard, en écho d'ailleurs aux Amours de Cassandre : 
Je voudrais bien pour alléger ma peine

Estre un Narcisse et elle une fontaine

Pour m'y plonger une nuit à séjour (Sonnet 20) formule: «in a kind of parody of poetic principles, the poem illustrates Narcissus' discovery that 'inopem me copia fecit' « (140). Le foisonnement qui régit la composition du poème shakespearien ne tient pas ses promesses de fécondité pour la déesse amoureuse et, tel le miroir de Narcisse, la richesse du reflet ne laisse présager que souffrance et privation. Ces multiples redoublements, que l'on peut tracer dans le poème, sont simultanément destructeurs, car ils reflètent une impuissante stérilité, et créateurs, puisqu'ils sont l'énergie même de la copia amoureuse et linguistique. À la vanité narcissique du reflet, du double, s'ajoute l'inanité des effets de la suasio vénusienne, autre Écho, qui fait retentir de sa plainte amoureuse les fonds des forêts. Un seul exemple : les fleurs de l'amour et les fleurs de la rhétorique ${ }^{23}$. La mort d'Adonis est évoquée deux fois dans le poème. D'abord par une analepse prophétique de Vénus :

An image like thyself [Adonis], all stained with gore,

Whose blood upon the fresh flowers being shed,

Doth make them droop with grief and hang the head. (664-666)

Puis par la description du narrateur :

No flower was nigh, no grass, herb, leaf, or weed,

But stole his blood, and seemed with him to bleed. (1055-1056)

Le déplacement, du corps de l'aimé, sanglant, aux fleurs, qui semblent saigner avec lui, sont une image de la métamorphose à l'œuvre qui offre, à un autre niveau, l'exemple de l'aporie de l'énergie créatrice puisqu'elle culmine dans l'expression de la mort. L'image s'inscrit certes dans la continuité thématique et narrative, mais elle dévoile aussi une intention rhétorique : par le biais de la réduplication copieuse, de fleur en fleur, pourraiton dire, les fleurs de rhétorique sont relues à la lumière du mythe diffus d'Écho et Narcisse. 
37 Finalement, l'accumulation d'images et d'arguments de Vénus, repris en écho par la voix narrative, oppose une forme d'obstacle à la vraisemblance du discours amoureux et concourt à recentrer la lecture sur la lettre du texte.

À la multiplicité des voix dans Les Vers d'Eurymédon et de Callirée, qui offrent une poétique éclatée et qui jouent des inventions du mythe pour introduire le souffle de la création ronsardienne, répondent les multiples échos de la rhétorique amoureuse de Shakespeare. Ronsard a toujours eu l'intention d'illustrer la créativité française en concurrence avec la tradition antique : la dialectique entre le discours « hérité », le discours d'autrui, celui du mythe, et l'expression de soi prend une résonance particulière puisque c'est par le biais $\mathrm{du}$ mythe que s'exprime la vision amoureuse du poète. L'on voit que, comme dans l'esthétique shakespearienne, le mythe n'est point ornement obligé, exemplum qui importe sa lecture allégorique «toute faite », mais que son utilisation est vivifiée par les modalités d'une insertion à plusieurs voix, donnant naissance à une glose originale.

L'effet de discontinu ainsi obtenu montre la liberté de l'écriture mythologique des auteurs. Cette liberté est commune à Shakespeare et à Ronsard, à trente ans d'intervalle, et c'est peut-être cela le dénominateur commun de l'usage du mythe en cette fin de siècle: il offre aux auteurs l'occasion d'exprimer leur voix grâce à un corpus vivant, quoique démembré, comme celui d'Orphée, chantre des amours de Vénus et Adonis dans le livre $\mathrm{x}$ des Métamorphoses, dont la lyre continue d'émettre des sons. Loin du raisonnement pur, le mythe permet de saisir intuitivement l'instabilité du sens, le foisonnement des figures, la variété vertigineuse, la mutabilité des formes ; il est comme les vents qui jouent sur la lyre d'Orphée : « [s]a lyre, [qui] à l'impulsion des vents mouvant les cordes, accordait harmonieusement avec le chant ${ }^{24} »$, comme le dit joliment Rabelais dans le Quart Livre.

\section{NOTES}

1. Les citations sont données à partir de l'édition suivante : Ronsard, CEuvres complètes, éd. Jean Céard, Daniel Ménager et Daniel Simonin, coll. «Pléiade », Paris, Gallimard, 1994. « Adonis », t. 2, p. 315-324. «Eurymédon », t. 1, p. 305-320. Pour le poème de Shakespeare, The Complete Sonnets and Poems, éd. Colin Burrow, coll. « The Oxford Shakespeare », Oxford, O.U.P., 2002.

2. Pour une traduction du poème de Shakespeare, voir William Shakespeare, Euvres complètes, édition bilingue, " Tragicomédies II, Poésies », coll. « Bouquins », Paris, Robert Laffont, 2002.

3. Clark Hulse (Metamorphic Verse: The Elizabethan Minor Epic, Princeton, Princeton U.P., 1981) dans son chapitre II, consacré à "Petrarchan Rhetoric ", se place dans la perspective de la querelle sur l'immoralité du texte poétique qui fait rage à la fin du siècle et s'intéresse à la réception par le public élisabéthain de poèmes proposant une rhétorique au service de l'exploration de tensions entre poésie morale et poésie sensuelle. Sans traiter d'une telle utilisation du mythe, il explore les différents niveaux de lecture qu'autorise la "grammaire » pétrarquiste.

4. Il existe aussi une œuvre d'Amadis Jamyn sur le même thème : Les CEuvres poétiques, livres I, III et IV (1575), éd. Samuel M. Carrington, Genève, Droz, 1978, p. 6-9. Confronter les deux textes est 
très intéressant car cela permet de mettre en évidence la radicale innovation mythologique de Ronsard. La vaine controverse qui s'interroge sur l'antériorité de l'œuvre de Jamyn par rapport à celle de Ronsard n'amène aucun indice probant tant les deux œuvres sont différentes.

5. Voir notamment de Marcel Raymond, «Sur les Amours d'Eurymédon et de Callirée », p. 59-74, dans De Jean Lemaire de Belges à Jean Giraudoux, mélanges d'histoire et de critique littéraire offerts à Pierre Jourda, Paris, Nizet, 1970.

6. On ne retrouve pas cela chez Jamyn : «Venus adonc qui veit l'heure bien opportune / banda son arc plié comme un croissant de lune », p. 134.

7. Clément Marot, Euvres poétiques, Paris, Garnier-Flammarion, 1973, p. 383. Épigramme LXII, «L'enfant Amour n'a plus son arc estrange, / Dont il blessoit d'hommes et cueurs et testes ;/ Avec celluy de Diane a faict change ; / Dont elle alloit aux champs faire les questes [...]. »

8. Cette méthode est activement explorée par Leonard Barkan, The Gods Made Flesh, Metamorphosis and the Pursuit of Paganism, New Haven et Londres, Yale University Press, 1986, et par Jonathan Bate: "the classical text provides a narrative framework into which the Elizabethan writer inserts elaborate arguments thus demonstrating his own rhetorical skills [...] the creative imitator interprets his source narrative partly by means of other narratives that lie both outside and inside, around and within, it " (Shakespeare and Ovid, Oxford, Clarendon Press, p. 50-51).

9. Cénée, fille d'Elatus, roi d'Arcadie, devint homme après son viol par Neptune et se consacra à la chasse (Mét. XII). Voir aussi Énéide VI.455. C'est un exemple proverbial : Érasme, Adage IV, i.

10. Sur cette notion et pour d'autres exemples, notamment dans The Rape of Lucrece, voir Agnès Lafont, Visages de Diane dans le théâtre élisabéthain (1560-1616), thèse soutenue en décembre 2003, Université Paul Valéry-Montpellier III, Introduction méthodologique et illustration dans la partie II.

11. Voir l'article éclairant de Guy Demerson, «Diane pour Ronsard: un mythe taciturne et toujours menacé » in Le Mythe de Diane en France au XVI siècle, éd. Jean-Raymond Fanlo et MarieDominique Legrand, Cahiers d'Aubigné, Albineana 14, Niort, 2002, p. 81-106, ici cité p. 99.

12. "L'exemple d'Acteon et du jeune Thebain / Qui virent et Diane et Pallas dans le bain, / Me devroient faire sage... » (op. cit., « Eurymedon », p. 315).

13. Voir, par exemple, Achilles Bocchi, Symbolicae Quaestiones (Bologne, 1555), 115, décrit un animal attaché, en bonne part, comme un emblème de désir contrôlé. Sir John Harington considère le coursier de Renaldo comme un symbole d'intempérance dans sa traduction de l' Orlando Furioso, canto 2. Pour plus de références, voir Clark Hulse, Metamorphic Verse: The Elizabethan Minor Epic, p. 166-167.

14. Voir aussi p. 319-322.

15. Voir les analyses de Yves Peyré qui oppose les amours de Vénus et Adonis à ceux de Mars et Vénus, Venus and Adonis, Paris, CNED, 2003, p. 122-123.

16. Ce que Guy Demerson appelle ces « bricolages où l'on coupe thèmes ou motifs d'une légende pour les recoller dans un autre thème » (« Diane chez Ronsard », p. 102).

17. Voir François Rigolot, Poésie et Renaissance, sur la façon dont du Bellay raille les conventions de la poésie amoureuse (notamment p. 270-271). Chez Shakespeare, le trait semble plus subtil mais c'est le même « dévoilement du désir charnel » (p. 272).

18. Deux articles de L'Esprit Créateur sont consacrés à ce poèmes : John C. Lapp, « Ronsard and La Fontaine: two versions of Adonis", p. 125-144, 1970 et surtout Donald Stone, Jr, "Ronsard, Rhetoric and Adonis », p. 183-8, 1972, qui signale l'incongruité de l'intervention de Mars dans le récit ovidien. [Références signalées par Guy Demerson, « Diane pour Ronsard », p. 92].

19. Demerson, op.cit., p. 93.

20. Voir Guy Demerson, La Mythologie classique dans l'œuvre de la Pléiade, Paris, Droz, 1971, p. 407.

21. Dans les Mét. d'Ovide, Narcisse meurt de langueur. Ici, il meurt noyé comme dans Le Viol de Lucrèce, 265-266.

22. Y. Peyré, op. cit., p. 140-158 pour les exemples de création poétique. 
23. Voir le travail de Perrine Galand-Hallyn, Le Reflet des fleurs. Description et métalangage poétique d'Homère à la Renaissance, Genève, Droz, 1994.

24. Ceci est la chute de la narration du mythe d'Orphée par Rabelais dans le Quart Livre: « Nous serions bien ébahis si c'étaient les tête et lyre de Orpheus. Car après que les femmes Threisses eurent Orpheus mis en pièces, elles jetèrent sa tête et sa lyre dans le fleuve Hebrus. Icelles par ce fleuve descendirent en la mer pontique jusques en l'île de Lesbos, toujours ensemble sur mer nageantes. Et de la tête continuellement sortait un chant lugubre, comme lamentant la mort de Orpheus; la lyre, à l'impulsion des vents mouvant les cordes, accordait harmonieusement avec le chant » (Rabelais, Quart Livre, ch. 55, Euvres complètes, Paris, Seuil, 1973, p. 731). Pour une analyse de ce passage, voir Rigolot, Poésie et Renaissance, p. 31 ; Cave, Cornucopia, p. 142.

\section{RÉSUMÉS}

L'Adonis (1564) et Les Vers d'Eurymédon et de Callirée (1573) de Ronsard et, vingt ans plus tard, le Venus and Adonis (1593) de Shakespeare révèlent une variété des registres, à laquelle fait écho une variété des genres. La liberté des auteurs face à la fiction mythologique tirée des Métamorphoses est de mettre en conversation le mythe ovidien avec l'expression pétrarquiste de l'amour. La fable semble alors porteuse d'un commentaire détaché sur l'expression convenue du tourment amoureux.

Ronsard's Adonis (1564) and Les Vers d'Eurymedon et de Callirée (1573), then twenty years later Shakespeare's Venus and Adonis (1593) are three mythological epyllia that share a variety of registers and a variety of genres. Both authors freely adapt the mythological material drawn from Ovid's Metamorphoses to make it question the Petrarchan love conventions. The fabula then functions as a detached comment on the well-known expression of love torments.

\section{AUTEUR}

\section{AGNÈS LAFONT}

Agnès Lafont est maître de conférences en littérature anglaise du XVI ${ }^{\mathrm{e}}$ siècle à l'université Paul Valéry de Montpellier, membre de l'Institut de recherches sur la Renaissance, l'âge Classique et les Lumières (UMR 5186 du CNRS). Elle a consacré sa thèse (soutenue en 2003) à l'étude du mythe de Diane dans la littérature élisabéthaine (1560-1616). Elle a publié plusieurs articles sur les réemplois mythologiques au théâtre. 